Objectives: To evaluate the feasibility and benefit of improving medical economics and disease activity outcomes in rheumatic patients through online consultation based on SSDM by rheumatologist.

Methods: The rheumatologists implemented the education and training programs on patients in using SSDM and assist the patients in downloading the SSDM mobile application. The SSDM includes doctors' and patients' interfaces. The patients' terminal includes serial self-assessments (DAS28, SLEDAI, HAQ), medication management, adverse events management and lab records. After data entry, data synchronizes to the mobile of the authorized doctor. On the basis of these data, the rheumatologists can accept the request from their follow-up patients and practice consultation through SSDM in the form of text or voice.

Results: From Feb 2015 to Jan 2019, 679 rheumatologists supplied 7,405 patients (RA $35 \%$, SLE $23 \%$, AS $9.5 \%$, gout $8.8 \%$, Sjogren syndrome $3.8 \%$, OA $3.4 \%$ and other $16.5 \%$ ) with 10,527 consultations. The consulting fee ranged from RMB 0 to 500 yuan (USD: RMB =1: 6.81) each in average of $78.10 \pm 45.12$ yuan, which match the registration fee in hospital. The total fee for consultations was 822,169 yuan RMB. $37 \%$ patients receiving online consultation lived in different cities from the rheumatologists. If the patients seek medical care in hospital, in addition to the registration fees and medical expenses, the mean cost of transportation, accommodation, and lost wages was $577.48 \pm 505.21$ (200 2,800 ) yuan. The total of cost for all patients would have been $6,079,135.00$ yuan RMB, which is 7.39 times more compared with the cost of online. Among 2,611 RA and 1,671 SLE patients with repeat self-evaluations who were followed up for over 90 days, the treat-to-target rate improved from $28 \%$ to $45 \%$ (DAS28<=3.2) and from $41 \%$ to $70 \%$ $(\mathrm{SLEDAl}<=4)$, respectively. Survey shows that satisfaction rate with the consultations is $100 \%$.

Conclusion: Through online disease management and consultations using SSDM, Chinese patients with rheumatic diseases enjoy good quality of care at lower cost with high satisfaction. Armed with data science, SSDM may supply the rest world with an option for reshaping the healthcare system.

Disclosure of Interests: None declared

DOI: 10.1136/annrheumdis-2019-eular.6565

\section{AB1237 COST OF ILLNESS AND QUALITY OF LIFE IN ANKYLOSING SPONDYLITIS PATIENTS TREATED WITH ADALIMUMAB IN CHINA}

Ya Xie, Liudan Tu, LV Qing, Yutong Jiang, Zetao Liao, Shuangyan Cao, Qiujing Wei, Jieruo Gu. the Third Affiliated Hospital of Sun Yat-sen University, Rheumatology, Guangzhou, China

Background: Ankylosing spondylitis (AS) is a chronic inflammatory disease which may lead to limited physical function, impaired quality of life and increased economic burden for society. There were many studies about the superior effects of biologic agents on symptom release, disease activity and functional remission in AS patients. However, studies on the economic burden and health-related quality of life of AS patients in China were spark.

Objectives: To access the cost of illness, work limitation and quality of life in active ankylosing spondylitis (AS) patients using adalimumab in China.

Methods: A prospective study was performed in 91 patients with active AS in China. Adult patients (aged $\geq 18$ years) fulfilled the $1984 \mathrm{New}$ York modified criteria of AS with the Bath Ankylosing Spondylitis Disease Activity Index (BASDAI) $\geq 4$ and $\mathrm{C}$ reactive protein (CRP) 6 were enrolled from rheumatology center from Jan 2017 to Aug 2017. All participants received adalimumab (40mg per 2 weeks) therapy and completed questionnaires about disease characteristics, quality of life, direct and indirect costs. Only patients with pay-work completed the Work Limitation Questionnaire (WLQ) to accesses the impact of chronic health conditions on job performance and productivity. Quality of life was measured using the Ankylosing Spondylitis Quality of life (ASQoL) and EuroQol-5 Dimensions (EQ-5D).

Results: A total of 91 patients with mean age of 30 years old $(87.8 \%$ males) and mean disease duration of 10 years received adalimumab treatment for 24 weeks. $78.02 \%$ of patients have a paid job with average work productivity loss of $28 \%$ measured by WLQ. The annual estimated costs of each patient were $\$ 35238.8$ while the direct cost accounted for $90.2 \%$ and the cost of medication accounted for $78.6 \%$. There were significant differences in change of ASQoL (change, $3.89[95 \% \mathrm{Cl}, 3.06$ to 4.71]; $P<0.0001$ ) and EQ-5D (change, -0.19 [95\% $\mathrm{Cl},-0.24$ to -0.31 ; $P<0.0001)$ scores from baseline and 24 weeks, with more improvements after therapy compared with baseline. Cost of illness was estimated as
\$21927.38 per quality-adjusted life year and \$15728.16 per BASDAI unit, respectively.

Conclusion: The cost of AS patients treated with adalimumab therapy was high in China and symptoms and QoL improved significantly after therapy.

Disclosure of Interests: None declared

DOI: 10.1136/annrheumdis-2019-eular.5781

\section{AB1238 PATIENTS' PERCEPTIONS OF SUPPORT PROGRAMS FOR THE TREATMENT OF CHRONIC INFLAMMATORY DISEASES}

Nelly Ziade, Dima Dgheim, Farid Stephan, Rita Slim, Khalil Honein. Saint-Joseph University, Beirut, Lebanon

Background: Adherence to therapy in chronic diseases such as inflammatory rheumatic diseases (CIRD), inflammatory bowel diseases (IBD) and psoriasis (Pso) is a major condition to achieve positive outcomes. Patient support programs (PSPs) were developed to improve the quality of care and enhance adherence to therapy.

Objectives: To evaluate the perception of patients treated for inflammatory chronic diseases towards PSPs.

Methods: All available PSPs were identified at the national level, and their services were classified into categories: financial, logistic, educa tional, and emotional support. Consecutive adult patients with CIRD, IBD and Pso, enrolled in a PSP for more than 3 months, were interviewed by a trained medical student. Demographic data, disease and treatment characteristics were collected at the physician's clinic. Global satisfaction was estimated using a 5-points Likert scale, adherence to treatment was measured by the Compliance Questionnaire for Rheumatology (CQR), PSPs services were classified according to their importance to the patient using a 5-points Likert scale. An open questionnaire identified the patients' perceptions qualitatively. Predictive factors of satisfaction were identified.

Results: Forty-seven patients were included in the study, 53\% were males, with a mean age of 49.8 years (SD 15.2) (Patients' characteristics in Table 1). The majority declared that the PSP was very useful $(95.7 \%)$ and were highly satisfied with the programs (97.9\%). Higher attributes were assigned, by decreasing order, to: financial (copay program, providing of free samples), logistic (hotline, refrigerating box), educational (educational material) and emotional support. Nursing services and telephone reminders were rated as least important (Figure 1). Most open comments gave higher appreciation to financial support (54\%), followed by education (38\%) and logistics (8\%). High appreciation of education was associated with lower age, type of treatment and PSP. Shorter treatment duration was associated with appreciation of educational material, emotional support and telephone reminders.

Conclusion: Patients were highly satisfied with PSP programs, and ranked the financial support as the most important followed by logistics whereas education, nursing services and telephone reminders were found less important. Lower age and shorter treatment duration were associated with higher appreciation of education and support.

\section{REFERENCES}

[1] Rubin D, Mittal M, Davis M, et al. Impact of a Patient Support Program on Patient Adherence to Adalimumab and Direct Medical Costs in Crohn's Disease, Ulcerative Colitis, Rheumatoid Arthritis, Psoriasis, Psoriatic Arthritis, and Ankylosing Spondylitis. J Manag Care Spec Pharm. 2017;23 (8):859-67

[2] Gangi A, Clewell J, Shillington A. The impact of patient support programs on adherence, clinical, humanistic, and economic patient outcomes: a targeted systematic review. Patient Preference and Adherence 2016:10 711725.

[3] Van den Bosch F, Ostor A, Wassenberg S, et al. Impact of Participation in the Adalimumab (Humira) Patient Support Program on Rheumatoid Arthritis Treatment Course: Results from the PASSION Study. Rheumatol Ther (2017) 4:85-96.

Table 1. Patients characteristics:

\begin{tabular}{|c|c|}
\hline $\mathrm{N}$ & 47 patients \\
\hline Disease, $\mathrm{N}$ (some patients have multiple diseases) & 18 \\
\hline - Inflammatory Bowel Disease & 17 \\
\hline - Axial Spondyloarthritis & 17 \\
\hline - Peripheral Spondyloarthritis & 13 \\
\hline - Rheumatoid Arthritis & 13 \\
\hline - Psoriasis & \\
\hline Age, mean (SD) & $49.8(15.2)$ \\
\hline
\end{tabular}


Male gender, $\mathrm{N}(\%)$

Current treatment, N (\%)

Anti-TNF alpha

Jak-Inhibitor

Anti-Integrin

Anti-IL12-23

Anti-IL17

Biologic treatment rank

$-1$

$-2$

$-3$

$-4$

5

Disease duration, years (SD)

Current treatment duration, years (SD)

Use of previous PSP, N (\%)

CQR score, mean (SD)

Importance of Patient Support Program Services

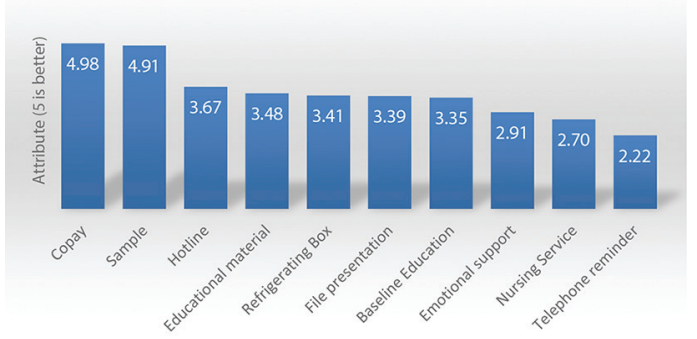

Abstract AB1238 Figure 1. Patient Support Program: Importance Assigned by the Patients to the different Services.

Disclosure of Interests: None declared

DOI: 10.1136/annrheumdis-2019-eular.6018

\section{Epidemiology, risk factors for disease or disease progression}

\section{AB1239 GENDER DIFFERENCE IN PULMONARY ARTERIAL HYPERTENSION, ASSOCIATED WITH CONNECTIVE TISSUE DISEASES}

Alexander Volkov, Natalia Yudkina, Ekaterina Nikolaeva, Evgeny Nasonov. VA Nasonova Research Institute of Rheumatology, Moscow, Russia, Moscow, Russian Federation

Background: Pulmonary arterial hypertension $(\mathrm{PAH})$ is a progressive fatal disease with a known gender dimorphism. However, data on gender differences at patients with pulmonary arterial hypertension, associated with connective tissue diseases (PAH-CTD), currently not enough.

Objectives: Therefore, this study aimed to investigate the role of gender in clinical future, hemodynamic data and survival PAH-CTD.

Methods: We examined the long-term prognosis of 97 consecutive $\mathrm{PAH}$ CTD patients, mean age 49 years ( 7 males and 90 females) diagnosed in our Institute from January 2009 to December 2018. The primary outcome was death. We used nonparametric analysis, Cox regression and the Kaplan-Meier method to assess variables obtained at baseline. All patients received $\mathrm{PAH}$-specific therapy according to the current recommendations.

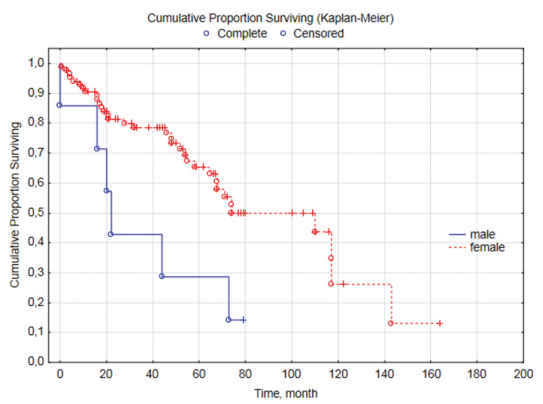

Figure 1
Results: We found that male patients had differences in diagnosis duration, level of creatinine, uric acid, RAP. We did not reveal differences in age, a functional class, a risk scale, and the majority clinical, tool and hemodynamic indicators. However, there were significant differences in survival. So, in male group survival was 21 [12; 51] months, in comparison in female group $74[48 ; 119]$ months, $p<0,02$. Thus, the male sex was an independent predictor of poor prognosis $(\mathrm{HR} 2.92[95 \% \mathrm{Cl} 1.21$ 7.03], $\mathrm{p}=0.018$ ) of PAH-CTD.

Conclusion: These results indicate that female PAH-CTD patients have better long-term prognosis than male, despite lack of many distinctions. It needs to be considered at outcome assessment.

Disclosure of Interests: Alexander Volkov : None declared, Natalia Yudkina: None declared, Ekaterina Nikolaeva: None declared, Evgeny Nasonov Speakers bureau: Pfizer, Inc., MSD, Novartis, AbbVie Inc., Celgen Corporation, Biocad, Janssen, UCB, Inc. DOI: 10.1136/annrheumdis-2019-eular.7912

\section{$\mathrm{AB} 1240$ \\ CAUSES AND ASSOCIATION OF DEATH IN SYSTEMIC LUPUS ERYTHEMATOSUS PATIENTS IN A TERTIARY REFERRAL UNIVERSITY HOSPITAL IN EGYPT}

Fatma Ibrahim Abdelrahman, Mohammed A. Mortada. Zagazig University, Rheumatology and Rehabilitation, zagazig, Egypt

Background: Systemic lupus erythematosus (SLE) is a chronic autoimmune disease with heterogeneous, multisystem involvements which can sometimes be severe and life threatening. Different causes of mortality have been described over different decades in SLE patients ${ }^{(1)}$

Objectives: To describe and analyze the causes of death in SLE patients and to identify the associated risk factors in Egypt.

Methods: A retrospective study was conducted on the SLE patients who died in the period from January 2014 to November 2019 in the Zagazig University hospitals. We collected demographic and laboratory data for all patients including; age of onset of SLE, age at death, disease duration, systems affected, last reported disease activity using the Systemic Lupus Erythematosus Disease Activity Index (SLEDAI), treatment received immunosuppressive regimen (dose and duration) and the cause of death.

Results: 41 SLE patients (33 female and 8 males) had died during period from January 2014 to November 2019 with mean age at onset

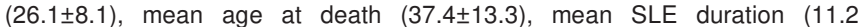
$\pm 6.7)$ and mean SLEDAI $(15.4 \pm 6.8)$. Majority of the dyed patients had lupus nephritis (87.8\%), (26.8\%) had neurophsycatric lupus, (14.6\%) had SLE vasculitis. (31.7\%) had APS, $(29.2 \%)$ had ILD, (14.6\%) had pneumonitis, (19.5\%) had carditis, and (14.6\%) had $\mathrm{PAH}$. All these patients were on a median dose of prednisolone $10(2.5-20) \mathrm{mg}$ daily with median of $10(4-24)$ years of steroids. 11 of these patients $(26.8 \%)$ had a family history of SLE. $21(51.2 \%)$ of them had received intravenous cyclophosphamide during their with mean cumulative dose of $(6.7 \pm 4.6$ gm, 16 (39\%) had received MMF, 5 (12.3\%) had received cyclosporine and $9(22 \%)$ had received azathioprine. As regard causes of death serious infection particularly pulmonary infection was the most common cause of death in 16 patients (39\%), followed by stroke in $8(19.5 \%$ ) patients, renal failure in $7(17.1 \%)$, pneumonitis in $3(7.3 \%)$, cancer in 3 (7.3\%), Disseminated Intravascular Coagulation (DIC) in $1(2.4 \%)$, Macrophage Activating Syndrome (MAC) in 1 (2.4\%), pancytopenia in 1 $(2.4 \%)$ and one $(2.4 \%)$ pregnant patient died from eclampsia in her third trimester with her fetus. The risk of death from serious pulmonary infec tions is higher in patients with ILD and pneumonitis $(p<0.000)$. The risk of stroke is higher in SLE patients with APS $(P=0.03)$ and neuro phsycatric lupus $(p=0.002)$.

Conclusion: pulmonary infections was the most common cause of death in SLE patients in a tertiary university hospital in Egypt.

\section{REFERENCES}

[1] Wu, X., Yang, M., Xie, Y. et al. Clin Rheumatol (2019) 38: 107. https://doi. org/10.1007/s10067-018-4259-z.

Disclosure of Interests: None declared

DOI: 10.1136/annrheumdis-2019-eular.6461 